\title{
Entrevista - Interview with Belinda Maia
}

Belinda, we know you as an enthusiast for technology and translation, and someone who did a lot to improve translator training so that new translators could also acquire digital competence. We would like to interview you personally, so that our readers can learn about your professional path and be motivated to read your papers later.

The first question is: what do you think was the best thing(s) you did in your (professional) life, and why?

First of all, let us confine this question to my professional life, particularly my work with training translators.

An outsider viewing my record would probably point to my involvement with the EMT - European Master's in Translation Network as my most important achievement in this area and, in many respects, I suppose it was. However, this involvement stemmed from my main objective as a university teacher, which was to provide students with the qualifications to earn their livings. This perspective developed over time and throughout the 40 years I worked at the Arts Faculty of the University of Porto (FLUP).

The main objective of the language departments for the 20 years after I started at FLUP in 1974 was to provide teachers of languages for the school system, especially in English. Translation was of little interest, until it became clear that the demand for teachers was drying up, and FLUP was forced to offer new job opportunities to their graduates. Fortunately, the need for professional translation was growing, particularly in scientific, technical, commercial areas.

My fascination with technology started simply enough with using corpora for research in linguistics, and went on to encouraging students to use everything the Internet had to offer. Then, when I asked experts questions about machine translation, I ended up in the LETRAC project (1997-8), an Alice in the Wonderland of language engineering, and my students found themselves using translation and localization software and doing terminology research with the help of the Linguateca project (2002-8). 
However, plenty of other people, both within academia and in the professional world soon realized how the translator's role was changing and how, as with many other areas, technology was key to this, and this led to the creation of the EMT Network referred to above. Like them, I have tried hard to keep up with the ongoing revolution in the language technology that affects not just translators but the general public as well.

On a broader level than translator training, I have always preferred applied to theoretical research, and believed that technology was there to forward such research, and that we should return the favour by cooperating with those who produce it. I believe that we can only really move forward and create useful results for research by embracing multi- and interdisciplinary cooperation.

\section{And what was your worst failure(s), and why?}

My worst failure is precisely my lack of success is persuading more people to move outside their restricted areas of research and experiment with the interdisciplinary cooperation referred to above. Although there were those who responded very positively to the challenges of creating a cross-disciplinary PhD course in Human Language Technologies, there were too many obstacles in our way, ranging from individual academic protectionism of certain disciplines or areas of research, to the bureaucratic ones of (inter)university administration.

\section{What would be your advice for people who want to become new transla- tors now?}

Before we start, let us be clear that the term 'translator' covers a wide variety of occupations, from a skill that is part of some other job, such as tour guide, secretary, teacher, or academic, to full-time professional translator working for a translation company or international organization. My advice here will be for those aiming for the professional side of this spectrum.

My first piece of advice is very practical: do some market research on the demand for your particular combination(s) of languages, and whether this demand exists locally or has to be sought globally. There is little point in 
thinking one can make a career out of translating directly between two languages that, in practice, use a 'bridge' language for most texts: for example, the European Commission and many commercial companies find it easier and cheaper to use an English or French version of the text as a bridge between, say, Finnish and Portuguese, than to employ a Finnish-Portuguese translator on the very few occasions when it is necessary. Similarly, the Portuguese translations of the Japanese writer Murakami are translated from the English translation, not directly from Japanese.

Then discover the direction in which you will be translating: if your mother tongue is not English, you will probably find that a lot of commercial, legal and even literary and journalistic work will be from English to your mother tongue, but that the demand from your language to another will be for local products, tourism, webpages, and academic work, usually into English. This is by no means an ideal situation, but I am being realistic.

So - make sure your language skills in both your mother tongue and the source languages are excellent and that you are competent in at least one bridge language.

If a market exists, be prepared to translate texts on any subject, particularly in the early stages of your career. This means you must learn how to specialize, however briefly, in any subject, and the Internet offers almost limitless resources for this, if you know how to use them wisely. There are many dictionaries, glossaries, terminology databases, monolingual and multilingual corpora, translation memories, machine translation, and sites that combine and mix these technologies, all freely available online. Use them, but learn to recognize quality in a resource. Wikipedia can be very useful for terminology, and a Google search will help you decide which of two terms is used in US or UK English, but using these resources sensibly is a learning process and takes time.

I cannot emphasize enough that the more you know how to use both translation and general information technology, the better. Translation memory software has provided standard tools for professional translators for well over a decade now, and you should learn how to work with as many versions as possible before you actually buy one (see Mark Shuttleworth's 
article in this volume). They are expensive, do not include ready made memories, and companies usually expect you to use their software + their memories. You should also be aware that the software is being constantly upgraded with new tools - and the upgrades are not free! For example, the more sophisticated brands now combine and reinforce the translation memories with machine translation techniques. Shuttleworth's article in this volume will explain some of these points in more detail but, as he says, most translation software doesn't work with Macs.

However, there are ways round this when you become more computer savvy, which is just as well as the Mac voice recognition software is excellent, according to those who have tried it. Believe it or not, you should learn - and teach your computer - to work with this software as, with experience, you - and especially you + all these tools - will one day work faster with your voice than with your fingers. And time is money.

If you want to be successful as a freelancer, make yourself as competent as possible with all kinds of software. For example, being able to translate / localize a webpage without technical help will accelerate the result for the client - but don't forget to charge for the expertise.

Do not deride or ignore machine translation (MT). Although it works better for some language pairs than others, there is a lot of paid work to be done post-editing the results. Find out and keep abreast of developments, as an intelligent interest in this area will help you advise clients as to whether their particular text will translate faster with MT + post-editing or not. Clients tend to be naïve as to MT's capabilities.

Although the following advice is given to most people starting in the job market today, it is particularly important here because I have often found that people planning to be translators think that it will be a way of working independently and without the hassle of cooperation with others. It is very important to develop your entrepreneurial and communicative skills, learn to work in teams - often in a company, make use of social networks and, specifically, get involved with the networks devoted to translation like Proz.com. Translators were among the first to use the Internet for mutual if virtual - help. Find out more. 


\section{And for those who teach translation?}

First of all, examine your own attitude to and reasons for teaching translation. Too many teachers of translation have little or no training or experience in professional translation and tend to use classes to foster their own interests in language teaching, linguistics, or literature. Even if these pedagogical objectives are what you are required to follow, find out what future your students can expect as translators and make sure you are competent to teach professional translation before you attempt to do so. For this, refer to what I have said above, make sure you understand the specific demands of the context in which you are working, adapt the types of translation work you do accordingly, acquire a working knowledge of translation software, and develop your skills in information mining well beyond that normally needed for the above-mentioned pedagogical objectives. Best of all, make sure you acquire some good hands-on professional experience working for a translation company. If you do all this and have good teaching skills, you will probably do a better job than professional translators with no teaching experience.

If you are also required to teach translation technology, you would be advised to read Mark Shuttleworth's article in this volume. However, you must bear in mind that, although the overall plan he presents is a good example, he is working at an English University. This means that his MA course will be one year only and not include the internships which most continental courses include as an essential part of their courses. The reason, of course, is the high fees that UK students have to pay in comparison to the fees in Europe, which tend to be much lower or even non-existent. European students, therefore, mind less about having to do (usually unpaid) professional internships of 2-6 months as a part of the course. Not only do the internships offer real experience, they often lead to jobs or allow students to overcome that demand by most employers - 'job experience'. As in other areas, internships are sometimes subject to abuse by employers, so universities should do their best to prevent this.

The other feature of UK courses is that they appeal to non-native speakers of English attracted by the possibility of improving their English. 
This explains why Shuttleworth was able to do the museum project he describes. In European universities, where most students will speak the language of their own country, translating such texts into other languages would put an unjustifiable burden on the teachers (presuming, of course, that they are native speakers of the target language). However, the emphasis on teamwork, with different roles like project manager, terminologists and translators has been part of standard EMT teaching for years and should be encouraged.

Shuttleworth's projects with machine translation and Termseeker are interesting and you can develop similar ones if you make the right contacts - try Kantan, the European Commission's MT projects or, if you are lucky, a language technology fan in your university's IT department.

However, although translation technology modules are essential in any MA course of this kind, one big problem, which Shuttleworth does not dwell on, is that of getting the teachers of translation modules to cooperate by applying the technology in their own classes. Some will, of course, understand the importance of this, but others will not.

A final word of warning - once a translation course exists in your university, beware of colleagues who approach you suggesting that your students might enjoy gaining (unpaid) 'experience' translating their engineering thesis and articles, or interpreting for their high-level medical conference. This work is difficult for top professionals with special subject qualifications.

\section{And for those who organize BA and MA progression in universities and high schools?}

Most universities today have to provide evidence of market research into the employability of the graduates from the courses they run, and, for translation courses, it is particularly important for them to follow the advice I have given students above, but at an institutional level. The universities must be aware of the technological side of translation and provide proper access to the necessary hardware, software and the Internet. Ideally they should also encourage cooperation between the translation course and the language 
technology interest of their IT department. Surprisingly enough, my experience as a member of the EMT board revealed that many university administrations and departments are hard to convince on these points. Their view of translation is often several decades out of date and the older professors in charge are somewhat technology shy as well. They should also be made aware that research that involves translation need not be confined to the Arts Faculty, but my answer below may help them to understand how.

Although translation has a role to play in the teaching of languages and the better understanding of the related cultures and literatures at both high school and BA level, I agree with those who believe that the training of professional translators is best left to MA level. This allows students from the more traditional modern languages courses to acquire a better grounding in the associated cultures, but it also attracts students with good language skills and first degrees in areas like law, science, or technology, who are much sought after by employers for their additional subject area knowledge.

\section{What is the theme(s) you would like to read/supervise a PhD about?}

My interests in $\mathrm{PhD}$ themes in this area are varied, and at present I am cosupervising theses on post-editing of machine translation, and the effect of the dominance of English on both the originals and translations of scientific and, particularly, legal language. All this research requires coordination between linguistic analysis at different levels based on specially designed corpora, translation, information technology, specialization in the subject field (the $2 \mathrm{PhD}$ students studying legal language have degrees in Law), and interaction with other translators for testing hypotheses (e.g. post-editing tools or the cognitive aspects of the translation process using keystroke and eyetracking technology).

In the future I would like to see research into the detection of plagiarism through translation. I have often intuited such plagiarism in student work, but the research needed to examine the subject properly would require both sophisticated computational linguistic analysis as well as coordination with plagiarism detection and data mining software. 


\section{Anything else you would like to say in an interview about translation and technology?}

I should like to emphasize that so much of all I have said above is relative to the 'local context' of the languages and demands for translation. Human language technologies are making great strides in the major languages of the world, but the lesser-used languages will depend heavily on the enthusiasm, not to mention funding, available. Translation software can be used with most languages but MT depends on greater resources. So, too, do the information resources described. For example, it is impossible to find the 'right term' online for a language in which an equivalent term has not yet been formulated by the expert in charge (if he or she exists).

I should also like to warn about over-enthusiasm for technology. I once listened to a computer engineer whose enthusiasm for translation memories had led him to create a BA course that promised to turn out competent translators using them. He believed the technology was the answer and that the students' competence in the languages used was of minor importance!

On the other hand, I would not agree with those who superciliously discard the help of technology to do literary translation. The information resources mentioned can still be invaluable, and translation memories may well help to retrieve previously translated material (authors, too, repeat their favourite expressions) and are useful for maintaining consistency when more than one translator is involved. Besides, a few months ago, I was made aware of a project for literary translators to create and share multilingual parallel corpora of literary works, together with a platform for exchanging ideas on how best to solve their problems.

Will MT ever be able to do literary translation? "Never say never". But for the time being literary translators may continue to work without being as dependent on technology as their colleagues in other areas. However, they should remember that it is calculated that about $95 \%$ of translation worldwide is non-literary, and they will probably always need other jobs or a private income in order to survive.

Finally, this is an interview and I have not supplied references. Should you need to check up on anything I have said, please don't hesitate to contact me. 